\title{
FINGER PRINT RECOGNITION USING DISCRETE WAVELET TRANSFORM
}

\author{
Thaiyalnayaki K. ${ }^{1}$, Syed Abdul Karim S. $^{2}$ Varsha Parmar P. ${ }^{3}$ \\ ${ }^{1}$ Department of Information Technology, Sri Venkateshwara College of Engineering Sriperumbudur, Chennai \\ Email: 'thaiyalnayaki@svce.ac.in, ${ }^{2}$ syedkarim_88@yahoo.com, ${ }^{3}$ varsha_casp@yahoo.com
}

\begin{abstract}
The most common approach for fingerprint analysis is using minutiae that identifies corresponding features and evaluates the resemblance between two fingerprint impressions. Although many minutiae point pattern matching algorithms have been proposed, reliable automatic fingerprint verification remains as a challenging problem. Finger print recognition can be done effectively using texture classification approach. Important aspect here is appropriate selection of features that recognize the finger print. We propose an effective combination of features for multi-scale and multi-directional recognition of fingerprints. The features include standard deviation, kurtosis, and skewness . We apply the method by analyzing the finger prints with discrete wavelet transform (DWT). We used Canberra distance metric for similarity comparison between the texture classes. We trained 30 images and obtained an overall performance up to $96 \%$.
\end{abstract}

Keywords: Wavelet transform, minutiae, finger print recognition, texture classification, multi-directional analysis.

\section{INTRODUCTION}

Finger prints are graphical ridge patterns present on human fingers, which, due to their uniqueness and permanence, are among the most reliable human characteristics that can be used for people identification. A common hypothesis is that certain features of the fingerprint ridges, called minutiae, are able to capture the invariant and discriminatory information present in the fingerprint image. A minutia detected in a fingerprint image can be characterized by a list of attributes that includes the minutia position, the minutia direction, and the type of minutia (ending or bifurcation). The representation of a fingerprint pattern thus comprises the attributes of all detected minutiae in a so-called minutiae set. By representing the minutiae set as a point pattern, the fingerprint verification problem can be reduced to a minutiae point pattern matching problem. Due to variations that may occur between two minutiae sets extracted from different impressions of the same finger, determining whether they indeed represent the same finger can be an extremely difficult problem. Both minutiae sets may suffer from false, missed, and displaced minutiae, caused by poor fingerprint image quality and imperfections in the minutiae extraction stage. Two fingerprints may be translated, rotated, and scaled with respect to each other and fingers may exert an unevenly distributed pressure across the acquisition sensor resulting in local nonlinear deformations due to the elasticity of the skin.
We use texture analysis using wavelet transform to overcome the above problems. The study of surface texture is commonly referred to as Surface Metrology. Texture is a fundamental characteristic in many natural images and also plays an important role in computer vision and pattern recognition. Texture analysis is an essential step for many image processing applications such as industrial inspection, document segmentation, remote sensing of earth resources, and medical imaging. For that reason, a great number of approaches to texture analysis have been investigated over the past three decades. Typical fingerprint surface consists of a range of spatial wavelengths with different amplitudes.

\section{PROPOSED APPROACH}

In this paper, we take the finger print images of ten persons and there are two rotated images for every person. Hence the database consists of 30 images. We train the system using these 30 images and when an input image is given to the system, it should recognize the person if there is a match. we propose a combination of three texture descriptors namely Standard Deviation, Kurtosis and Skewness. DWT is the transform used for analysis. Canberra distance metric is used for similarity estimation.

\section{A. 2D-Discrete Wavelet Transform}

A weakness shared by most of the texture analysis schemes is that the image is analyzed at one single-scale; a limitation that can be lifted by employing a multi-scale representation of the textures such as the 
one offered by the wavelet transform. Wavelets have been shown to be useful for texture analysis in literature, possibly due to their finite duration, which provides both frequency and spatial locality. The hierarchical wavelet transform uses a family of wavelet functions and its associated scaling functions to decompose the original signal/image into different sub bands. The decomposition process is recursively applied to the sub bands to generate the next level of the hierarchy.

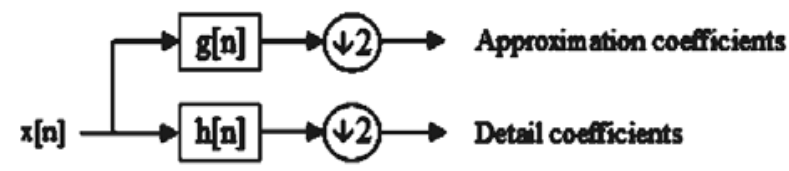

This shows one level DWT. At every iteration of the DWT, the lines of the input image (obtained at the end of the previous iteration) are low-pass filtered and high pass filtered. Then the lines of the two images obtained at the output of the two filters are decimated with a factor of 2. Next, the columns of the two images obtained are low and high pass filtered. The columns of those four images are also decimated with a factor of 2. Four new sub-images (representing the result of the current iteration) are generated. The first one, obtained after two low-pass filtering, is named approximation sub-image (or $L L$ image). The others three are named detail sub-images: $L H, H L$ and $H H$.

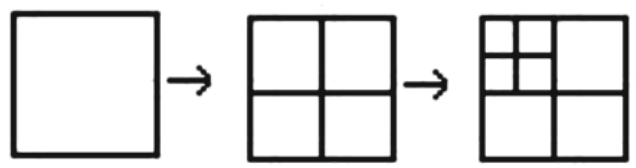

The $L L$ image represents the input for the next iteration.

In this paper we have used level 2 daubechies transform and only the second level $L L$ image is used for the analysis as that contains most of the important texture information. Daubechies deals with problems associated with JPEG compression and random additive noise.

\section{B. Feature Extraction}

Feature extraction is concerned with the quantification of texture characteristics in terms of a collection of descriptors or quantitative feature measurements, often referred to as a feature vector. The choice of appropriate descriptive parameters will radically influence the reliability and effectiveness of subsequent feature qualification through classification.

Algorithm for texture analysis and feature extraction with DWT:

1. Subject the gray scale texture image to a 2-level discrete wavelet transform decomposition

2. At each level, the wavelet transform decompose the given image in to three directional components, i.e., horizontal, diagonal and vertical detail sub bands in the direction of 0,45 and 135 respectively apart from the approximation (or) smooth sub band. For the second level LL sub-image compute the following three features.

\section{(i) Standard deviation}

The standard deviation of the image gives a measure of the amount of detail in that sub band.

\section{(ii) Kurtosis:}

It measures the peaked ness or flatness of the distribution and is given by

$$
k=\frac{1}{N} \sum_{i=1}^{N}\left(\frac{x_{1}=\mu}{\sigma}\right)^{2}
$$

where $\mu$ is the sample mean of the $N$ pixels within the image and ó is standard deviation

\section{(iii) Skewness}

Skewness is a measure of the asymmetry of the data around the sample mean.

$$
H=\frac{m_{3}}{m_{2}^{3 / 2}}=\frac{\frac{1}{n} \sum_{i=1}^{n}\left(x_{i}-\bar{x}\right)^{3}}{\left(\frac{1}{n} \sum_{i=1}^{n}\left(x_{i}-\bar{x}\right)^{2}\right)^{3 / 2}}
$$

where, $x_{i} s$ the ith value, $\bar{x}$ is the sample mean, $m_{3}$ is the sample thired central moment, and $m_{2}$ is the sample variance. Given samples from a population, the equation for the sample skewness $g 1$ above is a biased estimator of the population skewness. A distribution that is skewed to the left(the tail of the distribution is heavier on the right) will have a negative skewness. A distribution that is skewed to the right(the tail of the distribution is heavier on the left), will have a positive skewness. 
3. Compute the three features for original image also. Thus the length of the feature vector is 3 features ${ }^{*}\left[\left({ }^{*} 10\right)+\right.$ original image $]=93$ features.

\section{Training}

We define the texture class first as we use supervised classification method. Each person belongs to a separate class. Each class consists of three images of the finger print of a person at different orientations. In the training phase, feature set is formed using the proposed algorithm. For three images in each class, average of each feature is calculated and is stored in texture feature database. This feature is used for texture classification.
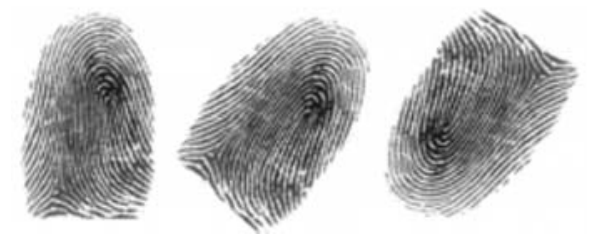

The above figure shows the finger prints of the same person at different orientations. The proposed algorithm can detect even if the test image is at some other orientation.

\section{Texture Classification}

In the texture classification phase, the texture feature set for the test sample $X$ is computed using the proposed algorithm. The feature database of texture classes $k$ prepared during training phase is used to compare the feature of test image. The distance metric can be termed as similarity measure. The distance between the texture classes stored in database and the test image is computed and used for classification. The test image is more similar to database class if the distance is smaller. The test image is recognized to belong to one of the classes of the database, if the smallest distance between the test image and texture classes is lesser than a threshold value. The class which gives the smallest distance is recognized as the person, whose finger print is given for testing. If the smallest distance is not lesser than the fixed threshold then the test image doesn't belong to any of the texture classes. If $\mathrm{N}$ is the number of features in feature set $f, f(x) j$ is the th texture feature of the test sample $X$ and $f(k)$ jis the th texture feature of $k$ th texture class in the database, then the Euclidean and Canberra distance metrics are described as below:
Euclidean

$$
d_{E}(k)=\sqrt{\sum_{j=t}^{N}\left[f_{j}(x)-f_{j}(k)\right]^{2}}
$$

Canberra

$$
d_{\text {can }}(k)=\sum_{j=1}^{N} \frac{\left|f_{j}(k)-f_{j}(x)\right|}{\left|f_{j}(k)\right|+\left|f_{j}(x)\right|}
$$

In Canberra distance metric, the individual feature components are normalized before finding the distance between the two images.

\section{E. Experimental Results}

The database consists of finger print images of ten individuals in three different orientations, not necessarily same for each individual. DWT is used as the analysis tool. Daubechies wavelet is used as the mother wavelet. Two level $\mathrm{db} 10$ is used as it gives high compression and removes random additive noise. All three features are calculated for each imageas shown in table 1. The average value of each feature for each individual is used for classification. This value is compared with the test image's features using Euclidean and Canberra distance measures. The classification performance is the rate of correct classification of surface textures and it is concluded the Canberra distance metric outperforms Euclidean distance.

\section{CONCLUSION}

This approach is very simple compared to minutia point pattern matching algorithm. It is robust as DWT is rotation invariant transform. It is found that the feature set comprising 93 features gives the overall performance of correct classification as $96.78 \%$. Performance can be increased by increasing the number of images for each class and by extending the feature set by adding features that do not vary widely with the orientation of the image but at the same time give values which can be used to uniquely classify the finger print classes. This method of feature extraction and surface texture classification will be useful for surface roughness evaluation in on line product quality monitoring. Industrial inspection is also a very popular field for using wavelets. Indeed, they are well suited to detect defects like scratches on a uniformtexture. 
Table 1 Classification Performance - DWT

\begin{tabular}{|c|c|c|c|c|c|c|c|}
\hline TD & \multicolumn{7}{|c|}{ Texture Descriptor [Number of Features] } \\
\hline Class & SD [31] & $\begin{array}{c}\text { Kurtosis } \\
\text { [31] }\end{array}$ & $\begin{array}{c}\text { Skewness } \\
{[31]}\end{array}$ & $\begin{array}{c}\text { SD + Kurtosis } \\
{[62]}\end{array}$ & \begin{tabular}{|c|} 
SD + Skewness \\
{$[62]$}
\end{tabular} & $\begin{array}{c}\text { Skewness + Kurtos } \\
\text { is [62] }\end{array}$ & $\begin{array}{c}\text { SD + Kurtosis + Skewness } \\
{[93]}\end{array}$ \\
\hline 1. & 80.00 & 82.00 & 83.00 & 83.33 & 83.33 & 80.00 & 86.67 \\
\hline & 90.00 & 89.09 & 83.33 & 86.67 & 86.67 & 90.00 & 96.67 \\
\hline & 96.67 & 100 & 83.33 & 100 & 83.33 & 96.67 & 96.67 \\
\hline 2. & 89.77 & 84.67 & 90.00 & 89.00 & 86.53 & 90.05 & 93.33 \\
\hline & 87.56 & 88.34 & 88.44 & 89.73 & 90.70 & 90.93 & 97.56 \\
\hline & 86.45 & 86.34 & 84.63 & 87.55 & 92.44 & 94.21 & 96.67 \\
\hline 3. & 85.22 & 87.54 & 87.49 & 90.34 & 97.55 & 90.29 & 98.89 \\
\hline & 89.36 & 85.33 & 87.44 & $86 . .95$ & 92.43 & 98.39 & 98.99 \\
\hline & 83.45 & 85.38 & 81.59 & 88.1 & 85.29 & 94.42 & 96.45 \\
\hline 4. & 84.54 & 85.98 & 83.54 & 89.41 & 90.00 & 92.67 & 96.73 \\
\hline & 81.37 & 82.44 & 88.90 & 89.45 & 91.23 & 95.34 & 96.77 \\
\hline & 81.33 & 80.00 & 89.89 & 9034 & 92.09 & 96.57 & 98.09 \\
\hline 5. & 83.34 & 87.34 & 84.22 & 88.33 & 89.34 & 89.90 & 93.23 \\
\hline & 83.60 & 87.00 & 89.34 & 89.39 & 90.00 & 93.45 & 95.78 \\
\hline & 84.95 & 87.88 & 83.23 & 89.90 & 90.00 & 90.02 & 92.32 \\
\hline 6. & 84.34 & 87.20 & 82.17 & 88.37 & 90.00 & 91.23 & 96.78 \\
\hline & 82.33 & 85.31 & 89.20 & 89.99 & 90.79 & 94.67 & 96.66 \\
\hline & 83.37 & 86.33 & 87.49 & 89.03 & 89.67 & 92.38 & 95.00 \\
\hline 7. & 87.39 & 90.00 & 98.00 & 98.99 & 99.08 & 99.38 & 99.89 \\
\hline & 88.34 & 88.00 & 83.90 & 89.90 & 90.56 & 93.79 & 94.98 \\
\hline & 83.89 & 87.33 & 88.00 & 89.89 & 90.40 & 91.11 & 95.70 \\
\hline 8. & 83.54 & 87.33 & 89.34 & 90.39 & 91.33 & 92.19 & 93.00 \\
\hline & 81.00 & 84.89 & 85.33 & 89.32 & 92.72 & 93.85 & 94.16 \\
\hline & 83.30 & 84.99 & 81.10 & 86.34 & 89.63 & 89.90 & 91.36 \\
\hline 9. & 82.33 & 85.64 & 81.08 & 86.37 & 89.90 & 91.0 & 95.34 \\
\hline & 86.34 & 87.50 & 88.00 & 87.90 & 90.30 & 92.07 & 93.00 \\
\hline & 80.00 & 82.87 & 81.34 & 84.39 & 86.32 & 89.97 & 90.39 \\
\hline 10. & 84.32 & 85.44 & 85.90 & 86.00 & 87.39 & 87.00 & 91.38 \\
\hline & 79.30 & 80.11 & 80.00 & 81.25 & 83.98 & 86.34 & 89.00 \\
\hline & 81.11 & 83.02 & 85.30 & 85.99 & 89.62 & 90.67 & 93.81 \\
\hline
\end{tabular}




\section{REFERENCES}

[1] ASME B46.1, Surface texture (Surface roughness, waviness and lay), 1995.

[2] Kingsbury N.G., "Image processing with complex wavelets," Phil. Trans. Roy. Soc. London A, vol. 357, pp.2543-2560, September 1999.

[3] Kingsbury N.G., "Complex wavelets for shift invariant analysis and filtering of signals," Journal of applied and computational harmonic analysis, Vol. 10, No.3, pp.234-253, May 2001.

[4] Zeng W., Jiang X., and Scott P., "Metrological characteristics of dual tree complex wavelet transform for surface analysis," Meas. Sci.Technol., 16, pp. 1410-1417, 2005.

[5] Canny J., "A computational approach to edge detection," IEEE Trans. on Pattern Analysis and
Machine Intelligence, PAMI-8, no. 6, pp. 679- 698, 1986.

[6] Gonzalez R.C., Woods R.E., and Eddins S.L., Digital Image Processing Using Matlab, 1st Indian Reprint, Pearson Education, 2004, ch. 7.

[7] Bhandari S.H. and Deshpande S.M., "Wavelets for surface Metrology," Accepted for presentation in international conference ACVIT, Nov. 2007

Thaiyalnayaki $\mathbf{K}$. is an on job researcher on signal processing, image security, VLSI signal processing, Content based image retrival, currently working as assistant professor in Sri Venkatehwara College of Engineering, Sriperumbudur and having about 12 years experience in teaching. 